\title{
Non-Fickian Transport Under Heterogeneous Advection and Mobile-Immobile Mass Transfer
}

\author{
Alessandro Comolli ${ }^{1,2,3}$ • Juan J. Hidalgo ${ }^{1,3}$. \\ Charlie Moussey $^{4} \cdot$ Marco Dentz $^{1,3}$ (D)
}

Received: 22 January 2016 / Accepted: 27 May 2016 / Published online: 8 June 2016

C Springer Science+Business Media Dordrecht 2016

\begin{abstract}
We study the combined impact of heterogeneous advection and mobile-immobile mass transfer on non-Fickian transport using the continuous-time random walk (CTRW) approach. The CTRW models solute transport in heterogeneous media as a random walk in space and time. Our study is based on a $d$-dimensional CTRW model that accounts for both heterogeneous advection and mass transfer between mobile and immobile zones, to which we also refer as solute trapping. The flow heterogeneity is mapped into the distribution of advective transition times over a characteristic heterogeneity scale. Mass transfer into immobile zones is quantified by a trapping rate and the distribution of particle return times. The total particle transition time over a characteristic heterogeneity scale then is given by the advective time and the sum of trapping times over the number of trapping events. We establish explicit integro-partial differential equations for the evolution of the concentration and discuss the relation to the multirate mass transfer approach, specifically the relation between the trapping time distribution and the memory function. We then analyze the signatures of anomalous transport due to advective heterogeneity and trapping in terms of spatial moments and first passage times or breakthrough curves. The behaviors for different disorder scenarios are analyzed analytically and through random walk particle tracking simulations. Assuming that advective mass transfer is faster than diffusive, we identify three regimes of distinct transport behaviors, which are separated by the characteristic trapping rate and trapping times. (1) At early times, we identify a pre-asymptotic time regime that is fully determined by advective heterogeneity and which is characterized by superlinear growth of
\end{abstract}

\footnotetext{
$凶$ Alessandro Comolli

alessandro.comolli@idaea.csic.es

1 Institute of Environmental Assessment and Water Research (IDÆA), Spanish National Research Council (CSIC), c/ Jordi Girona 18, 08034 Barcelona, Spain

2 Department of Geotechnical Engineering and Geosciences, Technical University of Catalonia (UPC), c/ Jordi Girona 1-3, 08034 Barcelona, Spain

3 Hydrogeology Group (CSIC-UPC), Barcelona, Spain

4 École Nationale des Ponts et Chaussées, Cité Descartes, av. Blaise Pascal 6, 77455 Champs-sur-Marne, France
} 
longitudinal dispersion. (2) For longitudinal dispersion, we identify an intermediate regime of strong superlinear diffusion. This regime is determined by the combined effect of advective heterogeneity and trapping. (3) At larger time, the asymptotic trapping-driven regime shows the signatures of diffusion in immobile zones, which leads to both sub- and superlinear dispersion. These results shed some new light on the mechanism of non-Fickian transport and their manifestation in spatial and temporal solute distributions.

Keywords Continuous-time random walk · Multirate mass transfer · Anomalous transport · Stochastic modeling

\section{Introduction}

Solute transport in highly heterogeneous porous media is characterized by features that do not conform to advection-dispersion models characterized by equivalent transport parameters. This has been observed from pore to field-scale under forced and natural flow conditions. The non-Fickian character is manifest for example in nonlinear growth of solute dispersion, forward and backward tails in spatial tracer distributions (Koch and Brady 1988; Adams and Gelhar 1992; Moroni and Cushman 2001; Levy and Berkowitz 2003; Holzner et al. 2015), and in the occurrence of heavy-tailed solute breakthrough curves and first passage time distributions (Hatano and Hatano 1998; Haggerty et al. 2000, 2001; Berkowitz et al. 2001; Becker and Shapiro 2003; Cortis and Berkowitz 2004; Gouze et al. 2008; Kang et al. 2015). Anomalous dispersion behaviors have been observed in a wide range of physical and biological systems (Metzler et al. 2014). Methods and models to explain and quantify such anomalous behaviors include moment equations (Neuman and Zhang 1990; Morales-Casique et al. 2006) and projector methods (Cushman et al. 1994), as well as space and time fractional advection-dispersion equations (Benson et al. 2000; Cushman and Ginn 2000; Schumer et al. 2003).

In this paper, we focus on the continuous-time random walk (CTRW) and multirate mass transfer (MRMT) approaches for the modeling of anomalous transport. Both approaches are similar in nature in the sense that anomalous transport features are related to distributions of characteristic mass transfer time scales which renders the governing equations for solute concentrations non-local in time.

The CTRW approach models transport in heterogeneous media through a random walk in both space and time. The time increment can be related to the advective or diffusive transition times over a characteristic heterogeneity scale as well as to the time a particle is trapped or immobilized. The CTRW framework developed by Scher and Lax (1973) based on the model of Montroll and Weiss (1965) has found broad applications for the modeling of historydependent dynamics in fluctuating and disordered systems ranging from the dispersion of light in disordered optical media (Barthelemy et al. 2008) to the movement of single molecules in living cells (Barkai et al. 2012), among many others (Klafter and Sokolov 2005). In the 1990s, Harvey Scher and Brian Berkowitz realized that the CTRW provides a natural framework for the understanding and quantification of anomalous transport in heterogeneous fractured and porous media (Berkowitz and Scher 1995, 1997). Since then the CTRW has been used as an upscaling and average transport framework to quantitatively understand flow and transport processes in geological media (Berkowitz et al. 2006) and has shed light on the origins and mechanisms of non-Fickian transport from the pore (Bijeljic and Blunt 2006; Cortis et al. 2004; de Anna et al. 2013; Kang et al. 2014; Holzner et al. 2015) to the Darcy (Dentz and 
Castro 2009; Le Borgne et al. 2008b; Edery et al. 2014) and catchment scale (Scher et al. 2002).

The MRMT approach is based on a multicontinuum concept (Barenblatt et al. 1960) that represents a highly heterogeneous porous or fractured medium by a mobile continuum and a suite of immobile continua, which communicate through linear mass transfer mechanisms such as diffusion or first-order rate processes (Maloszewski and Zuber 1985; Roth and Jury 1993; Haggerty and Gorelick 1995; Carrera et al. 1998; Haggerty et al. 2000). The medium heterogeneity is encoded in the memory function, which reflects the mass transfer mechanisms, diffusion or slow advection and linear kinetic adsorption reactions, for example, and the geometry and heterogeneity of the immobile regions (Zinn et al. 2004; Gouze et al. 2008; Willmann et al. 2008; Zhang et al. 2014). It has been shown that MRMT can be modeled within the CTRW framework through mapping the memory function onto the distribution of transition times (Dentz and Berkowitz 2003; Schumer et al. 2003; Berkowitz et al. 2008). More specifically, the trapping process can be modeled within the CTRW framework as a compound Poisson process (Margolin et al. 2003; Benson and Meerschaert 2009; Dentz et al. 2012, 2015). This means that trapping events occur at constant rate, which renders the number of trapping events during a given time interval a Poisson process. The distribution of trapping times can be related directly to the memory function of MRMT, in fact, it is given by its derivative. Advective heterogeneity and solute trapping into immobile zones represent two mechanisms that can lead to anomalous transport as expressed through non-Gaussian spatial and temporal distributions and nonlinear evolution of spatial moments (Haggerty et al. 2000; Becker and Shapiro 2003; Kang et al. 2015). It is often not clear how to separate these mechanisms and their impact on large-scale transport because of their similar manifestation in the transport behavior. These questions have been approached in Kang et al. (2015) and Dentz et al. (2015) in the analysis of solute breakthrough curves in radial tracer tests. Here we set up a CTRW model that represents explicitly heterogeneous advection and solute trapping in $d$-dimensional heterogeneous media, in order to systematically study the manifestation of these different mechanisms on spatial and temporal large-scale transport characteristics. This allows to discriminate between advective and trapping mechanisms and quantify them from observations of anomalous transport. Furthermore, the formulation of MRMT in terms of CTRW gives some new insight in the relation of the trapping process and the mass transfer processes encoded by the memory function.

The next section describes the general CTRW framework and the specific implementation of advective heterogeneity and solute trapping. It discusses the equivalence to the MRMT approach and analyzes the relation between memory function, trapping rate and the distribution of trapping times. Section 3 defines the observables that are analyzed, namely the center of mass and dispersion of a solute distribution as well as the distribution of first passage times. We also visualize spatial solute distributions in $d=1$ and $d=2$ dimensions. Section 4 studies the temporal evolution of the spatial concentration moments and the behavior of the first passage time distributions for different heterogeneity scenarios.

\section{Continuous-Time Random Walk}

The CTRW models particle movements in a heterogeneous environment as a random walk in space and time such that the particle position $\mathbf{x}_{n}$ and $t_{n}$ after $n$ random walk steps are given by the stochastic recursion

$$
\mathbf{x}_{n+1}=\mathbf{x}_{n}+\xi_{n}, \quad t_{n+1}=t_{n}+\tau_{n},
$$


where $\xi_{n}$ and $\tau_{n}$ are random spatial and temporal increments, respectively. The stochastic dynamics of the heterogeneous system are mapped into the joint probability density function (PDF) of the random space and time increment $\psi(\xi, \tau)$, which is at the heart of the CTRW approach. The particle distribution, or equivalently concentration distribution $c(\mathbf{x}, t)$, is given in terms of the space-time particle trajectories as

$$
c(\mathbf{x}, t)=\left\langle\delta\left[\mathbf{x}-\mathbf{x}_{n_{t}}\right]\right\rangle,
$$

where the angular brackets denote the average over all particles; $n_{t}=\sup \left(n \mid t_{n} \leq t\right)$ is the number of steps needed to reach time $t$. In order to derive the governing equations for $c(\mathbf{x}, t)$, we follow Scher and Lax (1973) and consider $R_{n}(\mathbf{x}, t)=\left\langle\delta\left(\mathbf{x}-\mathbf{x}_{n}\right) \delta\left(t-t_{n}\right)\right\rangle$, the joint density of particle position and times after $n$ random walk steps. Note that the space-time random walk (1) is a Markov process in the step number $n$. Thus, its density $R_{n}(\mathbf{x}, t)$ satisfies the Chapman-Kolmogorov equation

$$
R_{n+1}(\mathbf{x}, t)=\int \mathrm{d} \mathbf{x}^{\prime} \int_{0}^{t} \mathrm{~d} t^{\prime} \psi\left(\mathbf{x}-\mathbf{x}^{\prime}, t-t^{\prime}\right) R_{n}\left(\mathbf{x}^{\prime}, t^{\prime}\right) .
$$

Note that the particle distribution $c(\mathbf{x}, t)$ measures the probability that a particle is at position $\mathbf{x}$ at time $t$ for any number of random walk steps. Thus, we define now the quantity $R(\mathbf{x}, t)=$ $\sum_{n=0}^{\infty} R_{n}(\mathbf{x}, t)$, which denotes the probability per time that the particle has just arrived at a position $\mathbf{x}$ at time $t$ after any number of steps. It is now evident that $c(\mathbf{x}, t)$ is constituted by the number of particles that have just arrived at position $\mathbf{x}$ and the ones that have arrived at an earlier time $t^{\prime}$ and have not finished the transition to the next position in space, or in other words, the transition time to any other position $\mathbf{x}$ is larger than $t-t^{\prime}$. The latter is given by

$$
\Phi\left(t-t^{\prime}\right)=\int_{t-t^{\prime}}^{\infty} \mathrm{d} \tau \psi(\tau)
$$

where $\psi(\tau)=\int \mathrm{d} \mathbf{x} \psi(\mathbf{x}, \tau)$ is the transition time PDF. Thus, the governing equation for $c(\mathbf{x}, t)$ can be written as

$$
c(\mathbf{x}, t)=\int_{0}^{t} \mathrm{~d} t^{\prime} R\left(\mathbf{x}, t^{\prime}\right) \Phi\left(t-t^{\prime}\right)
$$

which is complemented by the equation for $R(\mathbf{x}, t)$ as given by summation of (3),

$$
R(\mathbf{x}, t)=R_{0}(\mathbf{x}, t)+\int d \mathbf{x}^{\prime} \int_{0}^{t} d t^{\prime} \psi\left(\mathbf{x}-\mathbf{x}^{\prime}, t-t^{\prime}\right) R\left(\mathbf{x}^{\prime}, t^{\prime}\right) .
$$

The initial condition of the CTRW is denoted by $R_{0}(\mathbf{x}, t)=\left\langle\delta\left(\mathbf{x}-\mathbf{x}_{0}\right)\right\rangle \delta\left(t-t_{0}\right)$. Equations (5) and (6) can be combined into the generalized Master equation (Kenkre et al. 1973)

$$
\frac{\mathrm{d} c(\mathbf{x}, t)}{\mathrm{d} t}=\int \mathrm{d} \mathbf{x}^{\prime} \int_{0}^{t} \mathrm{~d} t^{\prime} \mathcal{K}\left(\mathbf{x}-\mathbf{x}^{\prime}, t-t^{\prime}\right)\left[c\left(\mathbf{x}^{\prime}, t^{\prime}\right)-c\left(\mathbf{x}, t^{\prime}\right)\right],
$$


where the memory kernel $\mathcal{K}(\mathbf{x}, t)$ is defined in Laplace space (Abramowitz and Stegun 1972) as

$$
\mathcal{K}^{*}(\mathbf{x}, \lambda)=\frac{\lambda \psi^{*}(\mathbf{x}, \lambda)}{1-\psi^{*}(\lambda)}
$$

Here and in the following, the Laplace variable is denoted by $\lambda$, Laplace transformed quantities are marked by an asterisk.

Scher and Lax (1973) developed this theory based on the CTRW model of Montroll and Weiss (1965) to quantify stochastic transport in disordered solids. Since then, it has found a wide range of applications in the modeling of the history-dependent dynamics in fluctuating and disordered systems (Klafter and Sokolov 2005). Most notably, the CTRW framework has been used for the understanding and quantification of non-Fickian transport in heterogeneous fractured and porous media in the seminal works of Berkowitz and Scher (1995) and Berkowitz and Scher (1997). In this context, the transition time $\tau$ of a solute particle is related kinematically to the transition length $\ell=|\xi|$ through the particle velocity $v$ as $\tau=\ell / v$ as discussed in the following.

In the following sections, we will make use of this CTRW framework to model two distinct mechanisms that enhance non-Fickian transport. First, we will consider heterogeneous advection which arises because of the heterogeneity of the hydraulic conductivity field. The second process that we are going to discuss is the trapping of solutes that describes mass exchange between mobile and immobile phase, as well as linear kinetic adsorption reactions (Roth and Jury 1993; Haggerty and Gorelick 1995; Carrera et al. 1998).

\subsection{Heterogeneous Advection}

We consider now purely advective transport in a heterogeneous velocity field $\mathbf{u}(\mathbf{x})$. The particle trajectory is described by the advection equation

$$
\frac{\mathrm{d} \mathbf{x}(t)}{\mathrm{d} t}=\mathbf{v}(t)
$$

where $\mathbf{v}(t)=\mathbf{u}[\mathbf{x}(t)]$ denotes the Lagrangian particle velocity. The distance $s(t)$ and the time $t(s)$ travelled along a streamline can be written as

$$
\frac{\mathrm{d} s(t)}{\mathrm{d} t}=v[s(t)], \quad \frac{\mathrm{d} t(s)}{\mathrm{d} s}=\frac{1}{v_{s}(s)},
$$

where $\mathbf{v}_{s}(s)=\mathbf{v}[t(s)]$ and $v_{s}(s)=\left|\mathbf{v}_{s}(s)\right|$ is the velocity along the streamline. It has been shown for flow in pore and Darcy scale heterogeneous porous and fractured media media (Berkowitz and Scher 1997; Benke and Painter 2003; Le Borgne et al. 2008b; Kang et al. 2011, 2014, 2015; de Anna et al. 2013) that particle velocities measured equidistantly along particle trajectories form a Markov process. Thus, the streamwise velocities can be characterized by their steady state distribution and a transition probability (Le Borgne et al. 2008a). For distances larger than the correlation length $\ell_{c}$ along the streamline (Kang et al. 2015), subsequent particle velocities can be considered independent. Thus, by coarse graining the particle movement on a scale $\Delta s$ larger or equal to the correlation distance $\ell_{c}$, we can write the equations of motion of solute particle as

$$
\mathbf{x}_{n+1}=\mathbf{x}_{n}+\ell_{c} \boldsymbol{\omega}_{n}, \quad t_{n+1}=t_{n}+\frac{\ell_{c}}{v_{n}},
$$

where $\omega_{n}=\mathbf{v}_{n} /|\mathbf{v}|$ is the orientation of the streamwise velocity $\mathbf{v}_{n}=\mathbf{v}_{s}\left(s_{n}\right)$. We assume that the mean velocity is aligned with the 1-direction of the coordinate system. Thus, we consider 
$\omega_{n}$ as a random vector whose average is $\langle\boldsymbol{\omega}\rangle=\mathbf{e}_{1}$ where $\mathbf{e}_{1}$ is the unit vector in 1-direction. Berkowitz and Scher (1997) have shown that particle transport in random fracture networks can be described by a CTRW in terms of the PDF of streamwise particle velocities. The streamwise velocities $v_{n}$ are fully characterized by the one-point PDF $p_{v}(v)$. Under ergodic conditions $p_{v}(v)$ is related to the Eulerian velocity PDF through flux weighting [Dentz et al., unpublished]. Furthermore, it has been shown (e.g., Fiori et al. 2007; Cvetkovic et al. 2014; Edery et al. 2014) that low flow velocities are proportional to hydraulic conductivity, which allows to map the conductivity PDF into the Eulerian velocity PDF. The transition time is given by $\tau_{a}=\ell_{c} / v$. Thus, the joint PDF of transition length and transition time is

$$
\psi_{a}(\xi, \tau)=p_{\xi}(\xi) \psi_{a}(\tau), \quad \psi_{a}(\tau)=\frac{\ell_{c}}{\tau^{2}} p_{v}\left(\ell_{c} / \tau\right)
$$

It decouples because the constant transition length $|\xi|=\ell_{c}$ is constant. The PDF $p_{\xi}(\xi)$ of space increments has mean $\langle\boldsymbol{\xi}\rangle=\ell_{c} \mathbf{e}_{1}$. In the following, we consider $p_{\xi}(\boldsymbol{\xi})$ with a mean that is aligned with the 1-direction of the coordinate system, and isotropic variance such that the first and second moments are given by

$$
\left\langle\xi_{i}\right\rangle=\delta_{i 1} \ell_{c}, \quad\left\langle\xi_{i} \xi_{j}\right\rangle=\ell_{c}^{2} \delta_{i 1} \delta_{j 1}+\delta_{i j} \sigma^{2} .
$$

\subsection{Heterogeneous Advection and Trapping}

The MRMT approach considers solute transport under mass transfer between a single mobile zone and a suite of immobile zones. It has been used in the literature to model solute retardation due to slow advection (Willmann et al. 2008), diffusive mass exchange between mobile and immobile regions (fracture-matrix mass exchange, for example) (Maloszewski and Zuber 1985), as well as linear kinetic adsorption reactions (Villermaux 1987; Roth and Jury 1993), see also Haggerty and Gorelick (1995) and Carrera et al. (1998). The linear mass transfer processes quantified by the MRMT model are typically formulated within a Eulerian framework as a non-local advection-dispersion equation, or equivalently by a system of two coupled equations, an advection-dispersion equation for the mobile regions and first-order rate equations, or a diffusion equation for immobile mass transfer (Carrera et al. 1998). Alternative Lagrangian formulations include the works by Michalak and Kitanidis (2000) and Salamon et al. (2006).

Here, we model linear mass transfer processes within the CTRW framework (Margolin et al. 2003; Benson and Meerschaert 2009; Dentz et al. 2012, 2015; Gjetvaij et al. 2015). Mass transfer is represented by particle trapping events, which occur at constant rate $\gamma$. This implies that the spatial distribution of traps or immobile regions is uniform and that the number of trapping events increases with the time a particle is exposed to the traps. The condition of spatial uniformity may be relaxed if the distribution of traps can be characterized by a finite characteristic length scale. For fractal spatial distributions, this is different because of the lack of a representative elementary volume. Here trapping is represented by a constant rate process and thus the number $n_{\tau_{a}}$ of trapping events during a mobile transition of duration $\tau_{a}$ is Poisson distributed with mean $\left\langle n_{\tau_{a}}\right\rangle=\gamma \tau_{a}$,

$$
P\left(n, \tau_{a}\right)=\frac{\left(\gamma \tau_{a}\right)^{n}}{n !} \exp \left(-\gamma \tau_{a}\right)
$$

Each trapping event is associated with a trapping time $\tau_{f}$, which is distributed according to $p_{f}(\tau)$. Thus, for a given mobile time $\tau_{a}$, the total trapping time during a mobile step is thus 
given by

$$
\tau_{f, t}=\sum_{i=0}^{n_{\tau_{a}}} \tau_{f, i}
$$

It is a compound Poisson process whose density can be written in Laplace space as (Margolin et al. 2003)

$$
\psi_{f}^{*}\left(\lambda \mid \tau_{a}\right)=\exp \left(-\left\{\lambda+\gamma\left[1-p_{f}^{*}(\lambda)\right]\right\} \tau_{a}\right) .
$$

Thus, the total transition time is given by

$$
\tau=\tau_{a}+\tau_{f, t} .
$$

Its density reads in Laplace space as

$$
\psi^{*}(\lambda)=\psi_{a}^{*}\left(\lambda+\gamma\left[1-p_{f}^{*}(\lambda)\right]\right) .
$$

Note that the probability that the particle is trapped for a time longer than $t$ is given by

$$
\varphi(t)=\int_{t}^{\infty} \mathrm{d} t^{\prime} p_{f}\left(t^{\prime}\right) .
$$

As we will see in the following, the latter is equal to the memory function of the MRMT approach (Haggerty and Gorelick 1995; Carrera et al. 1998). Equivalently, the trapping time $\mathrm{PDF}$ is obtained for a given memory function as

$$
p_{f}(t)=-\frac{\mathrm{d} \varphi(t)}{\mathrm{d} t}
$$

We divide now the total concentration into a mobile and an immobile concentration,

$$
c(\mathbf{x}, t)=c_{m}(\mathbf{x}, t)+c_{i m}(\mathbf{x}, t) .
$$

The immobile and mobile concentrations are related as

$$
c_{i m}(\mathbf{x}, t)=\gamma \int_{0}^{t} \mathrm{~d} t^{\prime} \varphi\left(t-t^{\prime}\right) c_{m}\left(\mathbf{x}, t^{\prime}\right) .
$$

The right-hand side expresses the density of immobile particles by the probability per time that mobile particles get trapped at a given time $t^{\prime}, \gamma c_{m}\left(r, t^{\prime}\right)$, times the probability that the residence time in the immobile region is larger than $t-t^{\prime}$. Thus, at asymptotically long times, the ratio of the time averaged immobile and mobile concentrations is given by

$$
\lim _{t \rightarrow \infty} \frac{\bar{c}_{i m}(\mathbf{x}, t)}{\bar{c}_{m}(\mathbf{x}, t)}=\lim _{\lambda \rightarrow 0} \gamma \lambda^{-1}\left[1-p_{f}^{*}(\lambda)\right]=\gamma\left\langle\tau_{f}\right\rangle
$$

where $\left\langle\tau_{f}\right\rangle$ is the mean immobile time, if it exists, and the overline denotes the time average $\bar{c}(\mathbf{x}, t)=t^{-1} \int_{0}^{t} \mathrm{~d} t^{\prime} c\left(\mathbf{x}, t^{\prime}\right)$.

We can now express the Laplace transform of the total concentration $c(\mathbf{x}, t)$ in terms of the mobile and immobile concentrations as

$$
c_{m}^{*}(\mathbf{x}, \lambda)=\left[1+\gamma \varphi^{*}(\lambda)\right] c^{*}(\mathbf{x}, \lambda)
$$


Using the latter in the Laplace transform of (7) and using (18) in the definition of the memory kernel, we can write down the following governing equation for $c^{*}(\mathbf{x}, \lambda)$,

$$
\begin{aligned}
& \lambda c_{m}^{*}(\mathbf{x}, \lambda)+\gamma \lambda \varphi^{*}(\lambda) c_{m}^{*}(\mathbf{x}, \lambda)=c(\mathbf{x}, 0) \\
& +\int \mathrm{d} \mathbf{x}^{\prime} p_{\xi}\left(\mathbf{x}^{\prime}\right) \mathcal{K}_{a}^{*}\left(\lambda\left[1+\gamma \varphi^{*}(\lambda)\right]\right)\left[c_{m}^{*}\left(\mathbf{x}-\mathbf{x}^{\prime}, \lambda\right)-c_{m}^{*}(\mathbf{x}, \lambda)\right],
\end{aligned}
$$

where the purely advective memory kernel $\mathcal{K}_{a}^{*}(\lambda)$ is defined by (8) with $\psi^{*}(\lambda)$ replaced by $\psi_{a}^{*}(\lambda)$. Inverse Laplace transform of (25) gives

$$
\begin{aligned}
& \frac{\mathrm{d} c_{m}(\mathbf{x}, t)}{\mathrm{d} t}+\frac{\mathrm{d}}{\mathrm{d} t} \gamma \int_{0}^{t} \mathrm{~d} t^{\prime} \varphi\left(t-t^{\prime}\right) c_{m}\left(\mathbf{x}, t^{\prime}\right)=\int \mathrm{d} \mathbf{x}^{\prime} p_{\xi}\left(\mathbf{x}^{\prime}\right) \times \\
& \int_{0}^{t} \mathrm{~d} t^{\prime} \int_{0}^{t-t^{\prime}} \mathrm{d} \tau \mathcal{K}_{a}(\tau) \psi_{f}\left(t-t^{\prime}-\tau \mid \tau\right)\left[c_{m}\left(\mathbf{x}-\mathbf{x}^{\prime}, t^{\prime}\right)-c_{m}\left(\mathbf{x}, t^{\prime}\right)\right] .
\end{aligned}
$$

The right side can be read as follows. The flux toward $x$ is given by the probability that particles make a transition from $\mathbf{x}^{\prime}$ to $\mathbf{x}$ times the probability that during the time interval $\left(t-t^{\prime}\right)$ there is a mobile episode of duration $\tau$ and a trapping episode of duration $t-t^{\prime}-\tau$.

\subsubsection{Homogeneous Mobile Zone}

Constant advection in a homogeneous mobile zone is characterized in the CTRW framework by the exponential transition time

$$
\psi_{0}(t)=\frac{\exp \left(-t / \tau_{0}\right)}{\tau_{0}} .
$$

This implies that the memory kernel (8) reduces to a Dirac delta

$$
\mathcal{K}_{a}(t)=\frac{\delta(t)}{\tau_{0}}
$$

where $\tau_{0}$ is the characteristic mobile transition time. Using this kernel in (26), we obtain directly

$$
\begin{aligned}
& \frac{d c_{m}(\mathbf{x}, t)}{\mathrm{d} t}+\frac{\mathrm{d}}{\mathrm{d} t} \int_{0}^{t} \mathrm{~d} t^{\prime} \varphi\left(t-t^{\prime}\right) c_{m}\left(\mathbf{x}, t^{\prime}\right) \\
& =\int \mathrm{d} \mathbf{x}^{\prime} p_{\xi}\left(\mathbf{x}^{\prime}\right)\left[c_{m}\left(\mathbf{x}-\mathbf{x}^{\prime}, t\right)-c_{m}(\mathbf{x}, t)\right],
\end{aligned}
$$

because $\psi_{f}\left(t-t^{\prime} \mid 0\right)=\delta\left(t-t^{\prime}\right)$. In order to obtain the advective-dispersive MRMT formulation, we specify the mean and mean squared displacement as $\left\langle\xi_{i}\right\rangle=v \tau_{0} \delta_{i 1}$ and $\left\langle\xi_{i} \xi_{j}\right\rangle=v^{2} \tau_{0}^{2} \delta_{i 1} \delta_{j 1}+2 D \tau_{0} \delta_{i j}$, respectively, where $v$ is the transport velocity and $D$ is the dispersion coefficient. In the limit of $\tau_{0} \rightarrow 0$, the displacement distribution is sharply peaked about 0 such that we can expand $c_{m}\left(\mathbf{x}-\mathbf{x}^{\prime}, t\right)$ into a Taylor series for small $\left|\mathbf{x}^{\prime}\right|$. Thus, we obtain from (29)

$$
\frac{\partial c_{m}(\mathbf{x}, t)}{\partial t}+\frac{\partial}{\partial t} \gamma \int_{0}^{t} \mathrm{~d} t^{\prime} \varphi\left(t-t^{\prime}\right) c_{m}\left(\mathbf{x}, t^{\prime}\right)=-\left(v \frac{\partial}{\partial x_{1}}-D \nabla^{2}\right) c_{m}(\mathbf{x}, t) .
$$


Note terms of order higher than second in the Taylor expansion disappear in the limit $\tau_{0} \rightarrow 0$. The MRMT model described (30) has been frequently used in the literature (Haggerty and Gorelick 1995; Carrera et al. 1998; Haggerty et al. 2000) to quantify transport under mass transfer between mobile and immobile regions.

\subsubsection{Trapping Time Distributions}

The memory function $\varphi(t)$ encodes the mass transfer between mobile and immobile zones (Haggerty and Gorelick 1995; Harvey and Gorelick 1995; Carrera et al. 1998; Dentz and Berkowitz 2003; Dentz et al. 2011). For linear first-order mass exchange, it reflects the distribution of transfer rates between mobile and immobile regions (Haggerty and Gorelick 1995). For diffusive mass transfer, it is related to the PDF of particle return times to the immobile zone boundaries (Gouze et al. 2008). Thus it is determined by the geometries and the characteristic diffusion scales of the immobile regions. The formulation of MRMT in terms of the CTRW framework sheds some new light on the meaning of the memory function and its relation to the residence time $\operatorname{PDF} p_{f}(\tau)$. As outlined above, the memory function $\varphi(t)$ as defined in (19) is in fact the probability that a particle is trapped longer than a time $t$. The memory function here has the property $\varphi(0)=1$. Note that $\varphi(t)$ is often defined differently, namely such that its integral is normalized to 1 . Thus, in terms of the trapping time PDF, an alternative $\varphi_{a}(t)$ reads in terms of $p_{f}(\tau)$ as

$$
\varphi_{a}(t)=\frac{1}{\left\langle\tau_{f}\right\rangle} \int_{t}^{\infty} \mathrm{d} \tau p_{f}(\tau),
$$

which requires the existence of the mean residence time $\left\langle\tau_{f}\right\rangle<\infty$.

For first-order mass transfer, the memory function is given by the exponential

$$
\varphi(t)=\exp (-\alpha t)
$$

with $\alpha$ the release rate, and equivalently $\alpha^{-1}$ the characteristic trapping time. The corresponding trapping time PDF is given by

$$
p_{f}(t)=\alpha \exp (-\alpha t) .
$$

For diffusive trapping in slab-shaped semi-infinite immobile regions, the trapping time distribution is equivalent to the distribution of return times to the origin for a pulse input at the boundary. It is known that the return time PDF is not well defined in continuous space (Weiss 1994) but needs to be determined on a lattice, which here has the characteristic length $\ell_{g}$. In this case, the return time PDF, or equivalently the trapping time PDF is given by the inverse Gaussian

$$
p_{f}(t)=\frac{\exp \left(-\frac{\tau_{g}}{4 t}\right)}{t \sqrt{4 t / \tau_{g}}}
$$

where $\tau_{g}$ is a characteristic trapping time. It estimates the time that particles need to travel a distance $\ell_{g}$ by diffusion. Note that the trapping time PDF $p_{f}(t)$ decays for $t \gg \tau_{g}$ as $t^{-3 / 2}$. The PDF (34) is also termed Lévy distribution. The corresponding memory function is given by

$$
\varphi(t)=1-\frac{\Gamma\left(\frac{1}{2} \frac{\tau_{g}}{4 t}\right)}{\sqrt{\pi}},
$$


with $\Gamma(\beta, t)$ the incomplete Gamma function (Abramowitz and Stegun 1972). It behaves as $t^{-1 / 2}$ for $t \gg \tau_{g}$.

For diffusive trapping in slab-shaped immobile regions of finite size, on the other hand, the memory function can be written in Laplace space as (Harvey and Gorelick 1995; Carrera et al. 1998)

$$
\varphi_{a}^{*}(\lambda)=\frac{1}{\sqrt{\lambda \tau_{D}}} \tanh \left(\sqrt{\lambda \tau_{D}}\right),
$$

where $\tau_{D}$ is the characteristic diffusion scale. It is normalized to 1 and has an integrable singularity at $t=0$. It behaves as $\varphi(t) \propto t^{-1 / 2}$ for $t \ll \tau_{D}$. This means, however, that $\left\langle\tau_{f}\right\rangle=\varphi_{a}(0)^{-1}$ cannot be defined in terms of this memory function, for the reasons outlined above. Nevertheless, from the behavior of the memory function at times $t \ll \tau_{D}$, we deduce that the PDF of trapping times behaves as $p_{f}(t) \propto t^{-3 / 2}$, as in the case of the semi-infinite medium. For $t \gg \tau_{D}, p_{f}(t)$ is cut-off exponentially in the same way as the memory function $\varphi_{a}(t)$. In the following, our focus is on the pre-asymptotic behavior when the immobile zone has not yet equilibrated with the mobile zone, this means at times $t \ll \tau_{D}$. Thus, we will employ the trapping time PDF (34). It has been shown in Gouze et al. (2008) that the memory function $\varphi(t)$ may display behaviors that are different from the characteristic $t^{-1 / 2}$ if the immobile regions are heterogeneous. Thus, in order to account for diffusive trapping in heterogeneous immobile regions, we employ the more general Lévy stable distributions

$$
p_{f}^{*}(\lambda)=\exp \left[-\left(\tau_{g} \lambda\right)^{\delta}\right]
$$

where $0<\delta<1$. It behaves for $t \gg \tau_{g}$ as $p_{f}^{*}(t) \propto t^{-1-\delta}$. Note that $\tau_{g}$ is approximately the median time of the trapping time PDF. Note also that for $\delta=1 / 2$ (37) is the Laplace transform of the inverse Gaussian (34).

\section{Observables}

In the following, we define the spatial distribution and moments of the concentration distribution as well as first passage time distributions and present expressions for their quantification in the CTRW framework reported in the previous section.

\subsection{Spatial Moments}

The evolution of the spatial particle distribution is given by the generalized Master equation (7). In Fourier-Laplace space, we obtain the compact expression (Scher and Lax 1973)

$$
\tilde{c}^{*}(\mathbf{k}, \lambda)=\frac{1-\psi^{*}(\lambda)}{\lambda\left[1-\tilde{p}_{\xi}(\mathbf{k}) \psi^{*}(\lambda)\right]},
$$

where we used that the PDF of transition length and time decouples into $\psi(\mathbf{x}, t)=p_{\xi}(\mathbf{x}) \psi(t)$.

The Fourier transform is defined here by

$$
\begin{aligned}
& \tilde{c}(\mathbf{k}, t)=\int_{\mathbb{R}^{d}} \mathrm{~d} \mathbf{x} \exp (\mathbf{1} \mathbf{k} \cdot \mathbf{x}) c(\mathbf{x}, t), \\
& c(\mathbf{x}, t)=\int_{\mathbb{R}^{d}} \frac{\mathrm{d} \mathbf{k}}{(2 \pi)^{d}} \exp (-1 \mathbf{k} \cdot \mathbf{x}) \tilde{c}(\mathbf{k}, t) .
\end{aligned}
$$


Fourier transformed quantities are marked by a tilde, the wave vector is denoted by $\mathbf{k}$; the imaginary unit is denoted by 1 .

The first and second moments $m_{j}(t)$ and $m_{i j}(t)$ of the particle density $c(\mathbf{x}, t)$ can be expressed in Laplace space by using (38) as

$$
m_{i}^{*}(\lambda)=-\left.1 \frac{\partial \tilde{c}^{*}(\mathbf{k}, \lambda)}{\partial k_{i}}\right|_{\mathbf{k}=0}, \quad m_{i j}^{*}(\lambda)=-\left.\frac{\partial^{2} \tilde{c}^{*}(\mathbf{k}, \lambda)}{\partial k_{i} \partial k_{j}}\right|_{\mathbf{k}=0}
$$

The center of mass of the particle distribution is measured by $m_{i}(t)$, while its dispersion is quantified by the second centered moments

$$
\kappa_{i j}(t)=m_{i j}(t)-m_{i}(t) m_{j}(t) .
$$

By inserting (38) into (41) and using (13), one obtains for $m_{i}^{*}(\lambda)$ (Dentz et al. 2004)

$$
m_{i}^{*}(\lambda)=\delta_{i 1} \frac{\ell \psi^{*}(\lambda)}{\lambda\left[1-\psi^{*}(\lambda)\right]} .
$$

For the second moments, one obtains analogously

$$
m_{i i}^{*}(\lambda)=\frac{\left(\delta_{i 1} \ell^{2}+\sigma^{2}\right) \psi^{*}(\lambda)}{\lambda\left[1-\psi^{*}(\lambda)\right]}+\lambda m_{i}^{*}(\lambda)^{2} \delta_{i 1} .
$$

The off-diagonal components are zero by definition. These expressions form the basis for the calculation of the evolution of the spatial moments in the following.

\subsection{First Passage Time Distribution}

The first passage times of solute particles at a plane located at $x_{1}=x_{c}$ is defined here by

$$
\tau\left(x_{c}\right)=t_{n_{c}}, \quad n_{c}=\inf \left(n \mid x_{1, n} \geq x_{c}\right) .
$$

We consider here pulse injections at $\mathbf{x}=0$ at the time $t=0$. The generalization to different injection conditions is immediate, since it simply requires the superposition of the responses to pulses. The first passage time distribution (FPTD) is defined by

$$
f\left(t, x_{c}\right)=\left\langle\delta\left[t-\tau\left(x_{c}\right)\right]\right\rangle .
$$

Using (45), this expression can be expanded as

$$
f\left(t, x_{c}\right)=\sum_{n=0}^{\infty} f_{0}\left(n, x_{c}\right) p_{n}(t),
$$

where $f_{0}\left(n, x_{c}\right)=\left\langle\delta_{n, n_{c}}\right\rangle$ is the distribution of the numbers of steps needed to reach $x_{c}$ and $p_{n}(t)=\left\langle\delta\left(t-t_{n}\right)\right\rangle$ is the distribution of times $t_{n}$ after $n$ CTRW steps. For large values of $n$, $f_{0}\left(n, x_{c}\right)$ converges to an inverse Gaussian distribution that is well-peaked about the mean value $\left\langle n_{c}\right\rangle=\left\lceil x_{c} / \ell\right\rceil$, where the upper brackets denote the ceiling function. Therefore, we approximate

$$
f\left(t, x_{c}\right) \approx p_{\left\langle n_{c}\right\rangle}(t)
$$

Thus, we can develop explicit expressions for $f\left(t, x_{c}\right)$ along the lines of Dentz et al. (2015). In Laplace space, $p_{\left\langle n_{c}\right\rangle}(t)$ can be written as

$$
p_{\left\langle n_{c}\right\rangle}^{*}(\lambda)=\psi^{*}(\lambda)^{\left\langle n_{c}\right\rangle} .
$$


We define now $\psi^{*}(\lambda)=1-\Delta \psi^{*}(\lambda)$ in order to write

$$
p_{\left\langle n_{c}\right\rangle}^{*}(\lambda)=\exp \left(\left\langle n_{c}\right\rangle \ln \left[1-\Delta \psi^{*}(\lambda)\right]\right) \approx \exp \left[-\left\langle n_{c}\right\rangle \Delta \psi^{*}(\lambda)\right] .
$$

This expression is the basis for the derivation of the asymptotic behaviors of the FPTD under advective heterogeneity and solute trapping investigated in the following.

\section{Transport Under Heterogeneous Advection and Trapping}

Here we study transport under the combined action of heterogeneous advection and solute trapping in the framework of the CTRW model described in Sect. 2. We analyze the transport behavior for scenarios that correspond to different degrees of heterogeneity of the advective field and of the mass transfer processes. Namely, we will first present the case in which the mobile transition times follow an exponential distribution, which corresponds to a condition of weak advective heterogeneity as discussed in Sect. 2.2.1, while the distribution of trapping times is characterized by strong tailing characteristic for pre-asymptotic diffusive mass transfer. Secondly, we will consider the case of heterogeneous advection characterized by a strong tailing in the PDF $\psi_{a}(t)$ of the advective transition time PDF together with a broad distribution of trapping times $p_{f}(t)$, for which we employ the stable distributions (37).

Before proceeding to the analysis of the transport behaviors, we briefly discuss the characteristic time scales of the trapping process. The first time scale is represented by the inverse of the trapping rate $\tau_{\gamma}=\gamma^{-1}$. It has a double meaning. On the one hand, it represents the time at which, on average, particles undergo the first trapping event. On the other hand, it is the average time particles spend in the mobile phase. The second characteristic scale is given by the time at which the average time spent mobile $\tau_{\gamma}$ is equal to the average time spent immobile. A characteristic average trapping time after $n$ trapping events is given by $\left\langle\tau_{f}(n)\right\rangle \approx \tau_{g} n^{\frac{1-\delta}{\delta}}$, see "Appendix 1," while the average number of trapping events after time $t$ is given by $\left\langle n_{f, t}\right\rangle=\gamma t$. This means that the mean trapping time after time $t$ is given by

$$
\left\langle\tau_{f}(t)\right\rangle \approx \tau_{g}(\gamma t)^{\frac{1-\delta}{\delta}} .
$$

The mean trapping time increases as a power law of the overall travel time, which is a characteristic of a fractal process (Bouchaud and Georges 1990). By setting $\tau_{\gamma}=\left\langle\tau_{f}\left(\tau_{e}\right)\right\rangle$, we obtain for the time scale $\tau_{e}$

$$
\tau_{e}=\tau_{\gamma}\left(\gamma \tau_{g}\right)^{\frac{\delta}{1-\delta}}
$$

In the following, we investigate the temporal evolution of the spatial moments and the first passage time distributions in the light of these characteristic time trapping time scales.

\subsection{Weak Advective Heterogeneity-Multirate Mass Transfer}

The MRMT model has been extensively studied in the literature in terms of first passage times and breakthrough curves. Here we analyze the spatial aspects of the model in terms of the evolution of the spatial moments with respect to the characteristic trapping times discussed previously. For completeness, we also discuss the first passage time distributions in light of the parameters of the trapping model, specifically, the role of the trapping rate $\gamma$. The PDF $p_{f}(t)$ of trapping times is given by the Lévy distribution (34), while the distribution of advection times is given by the exponential (27). The advection scale $\tau_{0}$ represents the time at which particles have sampled full advective heterogeneity. Here we set $\tau_{0} \ll \tau_{\gamma} \ll \tau_{e}$. Thus, 

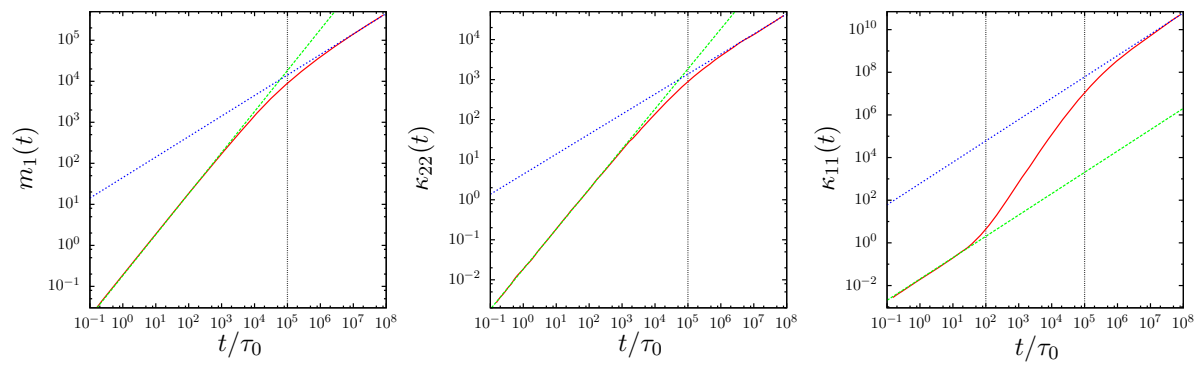

Fig. 1 (Left) center of mass position $m_{1}(t)$, (center) transverse variance $\kappa_{22}(t)$, and (right) longitudinal variance $\kappa_{11}(t)$, for $\delta=1 / 2, \gamma=10^{-2} \tau_{0}^{-1}$

we identify three time regimes, the pre-asymptotic time regime defined by $\tau_{0} \ll t \ll \tau_{\gamma}$, the intermediate time regime $\tau_{\gamma} \ll t \ll \tau_{e}$ and the asymptotic time regime $t \gg \tau_{e}$.

\subsubsection{Spatial Moments}

Figure 1 shows the behavior of the center of mass and the spatial variance of the particle distribution in the longitudinal and transverse directions obtained through numerical particle tracking simulations in $d=2$ spatial dimensions. We have studied the system under the condition of point-like injection at position $\mathbf{x}=0$ at time $t=0$. We observe that the mean in the longitudinal direction and the variance in the transverse direction exhibit very similar behaviors. In contrast, the behavior of the variance in the longitudinal direction is rather different as discussed in the following.

Pre-asymptotic regime $\tau_{0} \ll t \ll \tau_{e}$ For times $t>\tau_{0}$, particles have sampled the full advective heterogeneity and advection is homogeneous. Thus, the center of mass and the spatial variances scale linearly with time. While this behavior persists until the time scale $\tau_{e}$ for the center of mass and transverse dispersion, we observe an additional intermediate regime for the spatial variance in flow direction.

Intermediate regime $\tau_{\gamma} \ll t \ll \tau_{e}$ Due to the separation of trapped and advected particles, the solute distribution is elongated in flow direction, as illustrated in Fig. 2. In fact, we

Fig. 2 (Top panel) spatial particle density and (bottom panel) vertically integrated particle density at $t=4 \times 10^{2} \tau_{0}$ for $\delta=1 / 2, \gamma=10^{-2} \tau_{0}^{-1}$

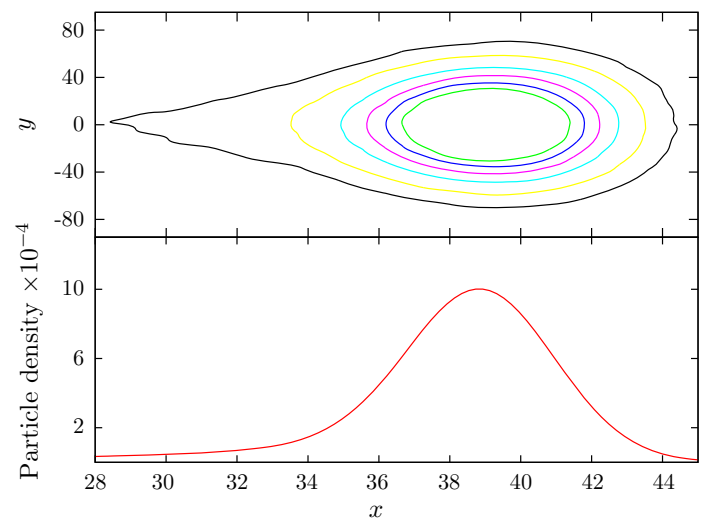


Fig. 3 First passage time distributions at $x_{c}=20 \ell_{c}$ for $\delta=1 / 2$ for (red solid line) $\gamma=10^{-1} \tau_{0}^{-1}$, (green dashed line) $\gamma=10^{-2} \tau_{v}^{-1}$ and (blue dotted line) $\gamma=10^{-3} \tau_{v}^{-1}$

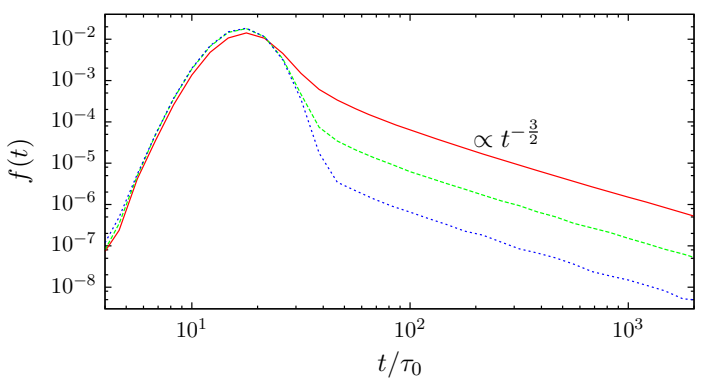

observe a strong increase of the longitudinal plume extension due to this chromatographic mechanism. This behavior sets on at the time $\tau_{\gamma}$ when the first particles get trapped and lasts until $\tau_{e}$, where the slope of $\kappa_{11}(t)$ starts decreasing.

Asymptotic regime $t \gg \tau_{e}$ At times larger than $\tau_{e}$, the time particles spend trapped is on average larger than the time mobile, which leads to a slowing down of particle motion. Trapping is the limiting process. As illustrated in Fig. $1, m_{1}(t)$ and $\kappa_{22}(t)$ cross over to their asymptotic subdiffusive behavior $t^{-1 / 2}$. The spatial variance in the longitudinal direction $\kappa_{11}(t) \propto t$ evolves linearly with time, which may suggest normal diffusive behavior. However, we have here rather an equilibrium between trapping and chromatographic stretching due to the fact that here the average time spent in the immobile zone given by (51) scales linearly with time. This means that the ratio between time spent in the mobile region and immobile traps is constant.

\subsubsection{First Passage Time Distribution}

Figure 3 shows first passage time distributions for three different values of the trapping rate $\gamma$ recorded at a control plane at $x_{c}=20 \ell_{c}$. These behaviors have been well known in the literature. We observe a characteristic advective peak at approximately $\tau_{p}=x_{c} / v$ with $v=\langle\xi\rangle / \tau_{0}$. At the peak time, the average time spent immobile is according to (51) $\left\langle\tau_{f}\left(t_{p}\right)\right\rangle \approx \tau_{g} \gamma t_{c}$. This explains the observation of slight retardation of the peak behavior for larger trapping rate $\gamma=10^{-1}$ compared to the smaller rates. At times larger than the peak time, the FPTD first decays exponentially fast before it shows the characteristic $t^{-3 / 2}$ tailing typical for matrix diffusion, see also "Appendix 3." The tailing is exclusively caused by particle release from the immobile traps, and it depends on the proportion of trapped versus mobile particles during the peak arrival times, which is approximately $\gamma$, see (22). Thus the onset of the power-law tail the FPTD is approximately $\gamma$ times the peak value of the FPTD as illustrated in Fig. 3.

\subsection{Heterogeneous Advection}

We study the combined impact of advective heterogeneity and solute trapping on the spatial particle distribution and first passage times. Velocity PDFs in highly heterogeneous porous media may be characterized by power-law behaviors at small velocities, while high velocities are rather unlikely (Berkowitz et al. 2006; Le Borgne et al. 2007; Edery et al. 2014). In order to account for these characteristics, we employ a Gamma distribution of transport velocities 


$$
\psi_{v}(v)=\left(\frac{v}{v_{0}}\right)^{\beta-1} \frac{\exp \left(-\frac{v}{v_{0}}\right)}{v_{0} \Gamma(\beta)} .
$$

We limit the range of the exponent to $1<\beta<2$ because advection is here the mobile transport mechanism and thus faster than trapping in immobile zones, which is characterized by the Lévy stable distribution (37). The advective transition time then is given by $\tau_{a}=\ell_{c} / v$. It is given by the inverse Gamma PDF

$$
\psi_{a}(t)=\left(\frac{t}{\tau_{v}}\right)^{-1-\beta} \frac{\exp \left(-\frac{\tau_{v}}{t}\right)}{\tau_{v} \Gamma(\beta)}
$$

where the scale $\tau_{v}=\ell_{c} / v_{0}$ marks the time after which particles start experiencing the spectrum of advective heterogeneity. It corresponds to the characteristic advection time over one correlation length. In the following, we analyze the evolution of the spatial moments and the behavior of the first passage times in light of the characteristic advection and trapping time scales.

\subsubsection{Spatial Moments}

Figure 4 shows the evolution of the center of mass as well as transverse and longitudinal spatial variances. We distinguish a pre-asymptotic regime set by $\tau_{v}$ and $\tau_{\gamma}$, a cross-over regime between $\tau_{\gamma}$ and $\tau_{e}$ and asymptotic regime for $t \gg \tau_{e}$ characterized by distinct temporal behaviors for all observables.

Pre-asymptotic regime $\tau_{v} \ll t \ll \tau_{\gamma}$ In this time regime, transport is dominated by advective heterogeneity. Particles sample the velocity variability, but the average number of trapping
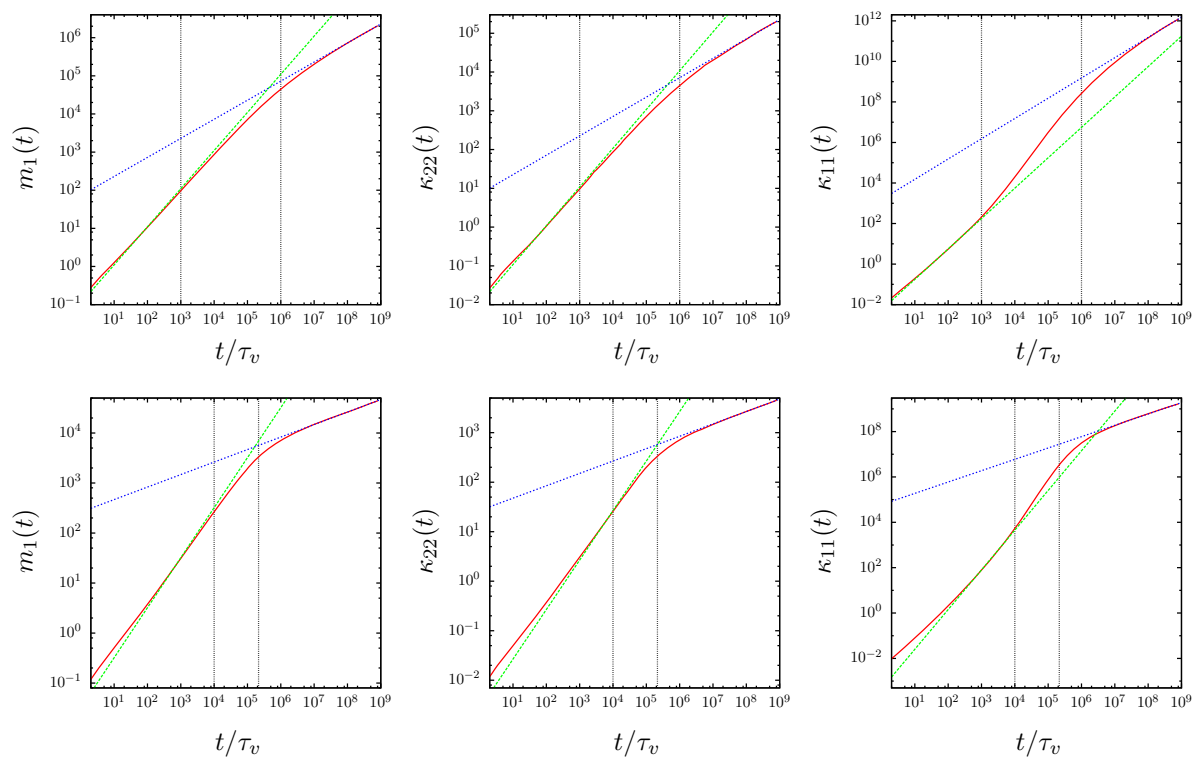

Fig. 4 (Left) center of mass position $m_{1}(t)$, (center) transverse variance $\kappa_{22}(t)$, and (right) longitudinal variance $\kappa_{11}(t)$, for (top row) $\beta=3 / 2, \delta=1 / 2, \gamma=10^{-3} \tau_{v}^{-1}$, and (bottom row) $\beta=5 / 4, \delta=1 / 4$, $\gamma=10^{-4} \tau_{v}^{-1}$. The vertical dashed lines denote (left to right) the corresponding $\tau_{\gamma}$ and $\tau_{e}$ 

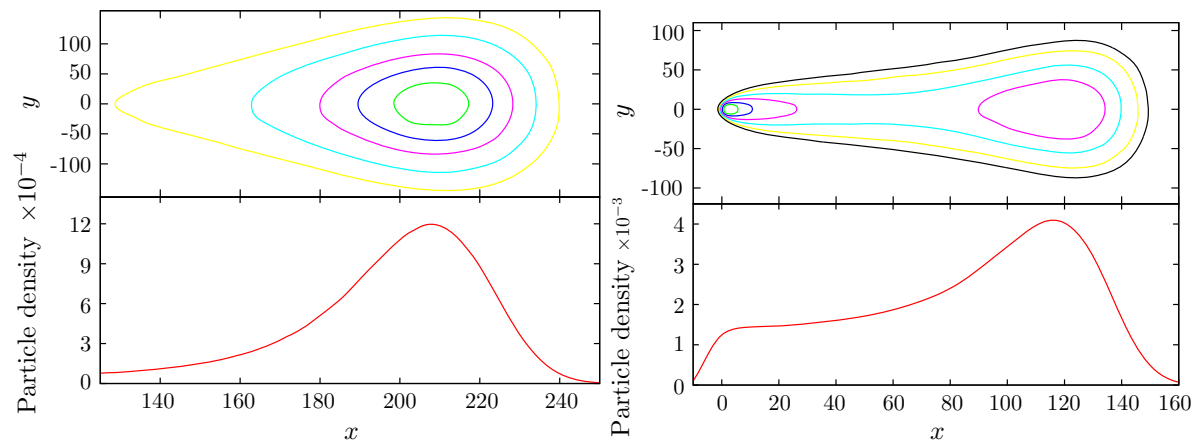

Fig. 5 (Top) spatial particle density and (bottom) vertically integrated particle densities at $t=4 \times 10^{3} \tau_{v}$ for $\gamma=10^{-3} \tau_{v}^{-1}$ and (left) $\beta=3 / 2, \delta=1 / 2$ and (right) $\beta=5 / 4, \delta=1 / 4$

events is smaller than 1 . Thus, $m_{1}(t) \propto t$ and $\kappa_{2}(t) \propto t$ evolve linearly with time, while the longitudinal variance scales as $\kappa_{11} \propto t^{3-\beta}$ (Dentz et al. 2004), see also "Strong Advective Heterogeneity" of Appendix.

Cross-over regime $\tau_{\gamma} \ll t \ll \tau_{e}$ Particles start experiencing the first trapping events. Thus, the center of mass position and transverse variance start deviating from the linear behavior and cross over to their asymptotic behavior. As in the previous scenario, we observe a the strong increase of the longitudinal variance $\kappa_{11}(t)$ due to the separation of mobile and immobile particles in flow direction. This chromatographic plume elongation illustrated in the spatial particle distribution shown in Fig. 5. We observe for both plumes a characteristic drop shaped form, while for $\beta=5 / 4$ and $\delta=1 / 4$ we observe a double peak behavior. This is a consequence of stronger particle trapping for decreasing $\delta$.

Asymptotic regime $t \gg \tau_{e}$ In this regime, the average time that particles spend in the immobile phase becomes larger than their average mobile times. Thus, the trapping process governs the transport behavior. While the center of mass position and transverse variance always show both subdiffusive growth as $\propto t^{\delta}$, the longitudinal variance scales as $\kappa_{11} \propto t^{2 \delta}$, see "Strong Advective Heterogeneity" of Appendix. This means that for $\delta=1 / 4$ the longitudinal variance scales as $\kappa_{11} \propto t^{1 / 2}$. The behavior is subdiffusive in flow direction because of the strong trapping. The average trapping time (51) increases here as $\left\langle\tau_{f}(t)\right\rangle \approx \tau_{g}(\gamma t)^{3}$; this means that the ratio of the total time spent mobile versus the average trapping time decreases as $\gamma t /\left\langle\tau_{f}(t)\right\rangle \propto t^{-2}$. The particles will eventually localize, which gives rise to the subdiffusive behavior. For increasing $\delta$, this is different. As discussed previously for $\delta=1 / 2$, $\left\langle\tau_{f}(t)\right\rangle \approx \tau_{g}(\gamma t)$. Thus, the ratio of total mobile time to average trapping time is constant, which here implies a linear growth of the longitudinal variance. For increasing $\delta>1 / 2$, the average immobile time decreases with increasing time. Thus $\kappa_{11}(t)$ evolves superlinearly, again due to the increasing distance between mobile and trapped particles.

\subsubsection{First Passage Time Distribution}

Figure 6 shows first passage time distributions for two different values of $\beta$ and $\delta$ and varying trapping rates $\gamma$. The peak arrival time is related to the mode of the Gamma velocity PDF, i.e., the most probable value of $v_{m}=v_{0} /(\beta-1)$ such that $\tau_{p} \approx x_{c} / v_{m}$. We distinguish again two time regimes. The early time regime is set by the peak time $\tau_{p}$ and characteristic time $\tau_{\gamma}$ for 

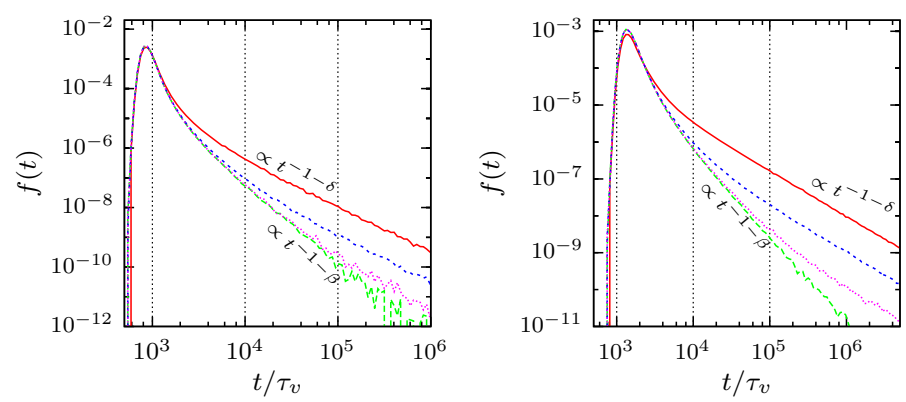

Fig. 6 First passage time distributions at $x_{c}=5 \times 10^{2} \ell_{c}$ for (left panel) $\beta=3 / 2, \delta=1 / 2$, and (right panel) $\beta=5 / 4$ and $\delta=1 / 4$ for (red solid line) $\gamma=10^{-3} \tau_{v}^{-1}$, (blue short dashed) $\gamma=10^{-4} \tau_{v}^{-1}$, (pink dotted) $\gamma=10^{-5} \tau_{v}^{-1}$ and (green long-dashed) $\gamma=0$. The advection time scale is $\tau_{v}=10^{-1}$. The black vertical dashed lines mark (left to right) $\tau_{\gamma}=10^{3} \tau_{v}, 10^{4} \tau_{v}$ and $10^{5} \tau_{v}$

the first trapping event to occur. For $\tau_{p} \ll \tau_{\gamma}$, the first passage time behavior in the regime $\tau_{p} \ll t \ll \tau_{\gamma}$ is dominated by the advective heterogeneity. The FPTD here scale as $f\left(t, x_{c}\right) \propto$ $t^{-1-\beta}$ (Berkowitz and Scher 1997), see also "Strong Advective Heterogeneity" of Appendix. For increasing time, particles start experiencing trapping events and the breakthrough curves cross over from the advective scaling toward the asymptotic scale $f\left(t, x_{c}\right) \propto t^{-1-\delta}$ in the asymptotic regime $t \gg \tau_{e}$.

\section{Summary and Conclusions}

We investigate the signatures of anomalous transport caused by heterogeneous advection and solute trapping. To this end, we use a CTRW model that distinguishes between the two disorder mechanisms. In this approach, the particle displacement is given by a characteristic velocity correlation scale. The transition time is composed of the advection time over this characteristic length and the total time particles spend trapped in immobile zones. The trapping process is modeled as a compound Poisson process. This means that trapping events are assumed to occur at a constant rate such that the number of trapping events during an advective transition is Poisson distributed. The total trapping time per step then is given by the sum of the trapping times over the number of trapping events. The distribution of trapping times can be related to the derivative of the memory of the MRMT approach, or directly derived as the distribution of return times to the boundaries of the immobile region. We derive the integro-differential equation governing the evolution of the total concentration as well as the mobile concentration for this scenario of coupled heterogeneous advection and solute trapping. For weak heterogeneity, we discuss the equivalence with the MRMT approach and study the relation between the memory function of MRMT and the distribution of trapping times. The CTRW model is formulated for $d$-spatial dimensions, our analysis of the transport behaviors is for illustration in $d=2$.

In order to identify the signatures of advective heterogeneity and solute trapping on largescale transport, we study the temporal evolution of the center of mass and dispersion of the particle distribution as well as the distributions of first passage times for different disorder scenarios. As we focus on aspects of anomalous transport, we consider stable distributions for the trapping times such that $p_{f}(t) \propto t^{-1-\delta}$ with $0<\delta<1$ and Gamma distributions of 
velocities which behave at small velocities as $p_{v}(v) \propto v^{\beta-1}$ with $1<\beta<2$. This gives rise to a distribution of advective transition times $\psi_{a}(t) \propto t^{-1-\beta}$.

We identify two characteristic time scales related to the trapping process, which set regimes of distinct transport behaviors. The first time scale is given by the inverse trapping rate, $\tau_{\gamma}=\gamma^{-1}$. It denotes the characteristic time after which a particle is trapped for the first time, as well as the mean time the particle is mobile. The second characteristic scale $\tau_{e}$ measures the time at which the particle has spent in average more time trapped than mobile. This means, for times larger than $\tau_{e}$ the transport behavior is dominated by the particle traps rather than by advection in the mobile zones. This time scale is given by $\tau_{e}=\tau_{\gamma}\left(\tau_{g} \gamma\right)^{\frac{\delta}{1-\delta}}$ with $\tau_{g}$ the median trapping time.

For weak heterogeneity, transport is Gaussian both in terms of spatial and temporal distributions for times smaller than $\tau_{\gamma}$ and larger than the characteristic advection time scale. For times larger than $\tau_{\gamma}$, the spatial distribution takes on a clearly non-Gaussian elongated drop shape as a consequence of the separation of mobile and trapped particles. This is reflected quantitatively in the evolution of the longitudinal cumulant, which increases superlinearly until the begin of the asymptotic time regime in which solute trapping starts dominating and slows down the rapid particle separation. The center of mass position and transverse cumulant are not affected by this chromatographic separation and evolve linearly until trapping dominates, from where on the behaviors become subdiffusive due to strong particle retention. These behaviors are also reflected by the first passage time distributions, characterized by an advective peak and a heavy tail due to particle trapping.

For strong heterogeneity, which is characterized by a high probability of low velocities, or long advective transition times, the behavior is qualitatively similar. Here we observe anomalous advection-dominated transport behavior for times that are larger than the characteristic advection, which is the time after which particle start sampling the advective heterogeneity. For times $t>\tau_{\gamma}$, we observe a similar chromatographic elongation due to the separation of mobile and immobile particles, which gives rise to a superlinear increase of the longitudinal cumulant. The increase is less drastic than in the case of weak heterogeneity because particles have in average lower velocities, which weakens the chromatographic effect. In fact, for decreasing $\delta$, this means stronger trapping, a secondary peak in the tail of the particle distribution forms because advection is efficient in the separation of trapped and advected particles. For times larger than $\tau_{e}$, trapping dominates and the longitudinal dispersion behavior is the same as in the case of weak heterogeneity. Again advective particle separation is not affecting the evolution of the center of mass position and transverse spreading. They both evolve linearly in time until they cross over to their asymptotic behavior on the time scale $\tau_{e}$, from which on trapping dominates. The first passage time distributions are characterized by two regimes which are set by the peak arrival time $\tau_{p}$ and the characteristic time $\tau_{\gamma}$ from the first trapping event. If both scales are well separate, we observe advection-controlled tailing behavior for $t \gg \tau_{p}$ and a cross-over to the trapping-controlled long-time behavior for $t \gg \tau_{e}$.

In conclusion, we have identified and quantified characteristic behaviors of spatial and temporal particle distributions as a response to advective heterogeneity and solute trapping. These results shed some new light on the manifestations of different heterogeneity mechanisms on large-scale transport, which may aid in the their identification from large-scale data and for the quantification of the pertinent heterogeneity parameters.

Acknowledgments The support of the European Research Council (ERC) through the project MHetScale (617511) is gratefully acknowledged. 


\section{Appendix 1: Trapping Time Scales}

The mass exchange process exhibits two distinct time scales. The first one is given by the inverse of the trapping rate $\tau_{\gamma}=\gamma^{-1}$. This time scale represents the time at which, on average, particles undergo the first trapping event. Moreover, it is also the average time that the particles spend in the mobile phase. For times larger than $\tau_{\gamma}$, advection ceases to be the only process that governs transport.

The second time scale represents the moment at which particles start spending on average more time trapped than in the mobile phase. In order to determine this scale, we need to compare the average time spent mobile, which is given by $\tau_{\gamma}$ to the average time spent immobile at a given time $t$. Note that the average trapping of the stable distribution (37) does not exist. Thus, in order to determine the characteristic trapping time after $n$ trapping event, we first consider the mean number $v\left(t_{f}\right) \equiv\left\langle n_{t_{f}}\right\rangle$ of trapping times needed to arrive at a given total trapping time $t_{f, n}=\sum_{i=1}^{n} \tau_{f, i}$, which is given by the renewal theorem as

$$
v\left(t_{f}\right)=1+\int_{0}^{t_{f}} \mathrm{~d} t^{\prime} v\left(t_{f}-t^{\prime}\right) p_{f}\left(t^{\prime}\right) .
$$

This equation is solved for the Laplace transform of $v\left(t_{f}\right)$ as

$$
v^{*}(\lambda)=\frac{1}{\lambda} \frac{1}{1-p_{f}^{*}(\lambda)} .
$$

Using the stable distributions (37) for small $\lambda \tau_{g}$ gives $\nu^{*}(\lambda) \approx \tau_{g}\left(\lambda \tau_{g}\right)^{-1-\delta}$, from which we obtain in time

$$
v\left(t_{f}\right) \approx\left(\frac{t}{\tau_{g}}\right)^{\delta} .
$$

The latter gives us a relation between the total trapping time and number of trapping events. We can use this relation to define a total mean trapping time as a function of step number by setting $v\left(\left\langle t_{f}\right\rangle\right)=n$, which gives $\left\langle t_{f}(n)\right\rangle=\tau_{g} n^{1 / \delta}$. Thus an average trapping time after $n$ steps is simply $\left\langle\tau_{f}(n)\right\rangle=\left\langle t_{f}(n)\right\rangle / n$, which gives

$$
\left\langle\tau_{f}(n)\right\rangle=\tau_{g} n^{\frac{1-\delta}{\delta}} .
$$

Notice that the latter is not strictly an average trapping time because for the stable distributions (37) the mean does not exist. It is rather a characteristic trapping time after $n$ trapping events.

\section{Appendix 2: Moments of the Spatial Distribution}

\section{Weak Advective Heterogeneity}

In the following, we will derive the expressions for the scaling of the moments and the variance of particle displacements in both the longitudinal and transverse directions in the pre-asymptotic and in the asymptotic time regimes. In particular, we will consider all the possible cases corresponding to different choices of the distribution of trapping times. For the derivation of the moments, we will make use of the inverse Gamma distribution for $\delta$ in 
$(1,2)$ and of stable distribution for $\delta$ in $(0,1)$. This choice has as a consequence the fact that the Laplace transform of the distribution of immobile times in the asymptotic limit $(\lambda \rightarrow 0)$ can be approximated for $\lambda \tau_{g} \ll 1$ as

$$
p_{f}^{*}(\lambda) \approx 1-\left(\lambda \tau_{g}\right)^{\delta} .
$$

By inserting Eq. (59) into Eq. (18), we get the expression for the distribution of transit times

$$
\psi^{*}(\lambda) \approx \frac{1}{1+\lambda \tau_{0}+\gamma \tau_{0}\left(\lambda \tau_{g}\right)^{\delta}}
$$

From Eq. (60), we identify the trapping time scale $\tau_{e}$ as follows. The second term in the denominator dominates for $\lambda \ll \gamma\left(\gamma \tau_{g}\right)^{\frac{\delta}{1-\delta}}$ and analogously for times $t \gg \tau_{\gamma}\left(\gamma \tau_{g}\right)^{\frac{\delta}{\delta-1}} \equiv \tau_{e}$.

We first derive the scalings of the moments in the pre-asymptotic time regime. Under this conditions, the PDF of transition times can be expanded and approximated with

$$
\psi^{*}(\lambda) \approx 1-\lambda \tau_{0}
$$

By inserting Eq. (61) into the equations for the first moment (43) and for the second moment (44) in this time regime and by considering the leading term in $\lambda$, we obtain

$$
m_{1}^{*}(\lambda) \propto \lambda^{-2}, \quad m_{11}^{*}(\lambda) \propto \lambda^{-3}+\lambda^{-2}, \quad m_{22}^{*}(\lambda) \propto \lambda^{-2} .
$$

We recall that the first moment in the transverse direction is always null. By applying the Tauberian theorems, we calculate the scaling in time from the expressions in the Laplace domain of Eq. (62). Thus, we find

$$
m_{1}(t) \propto t, \quad m_{11}(t) \propto t^{2}+t, \quad m_{22}(t) \propto t .
$$

These results are valid for $\delta \in(0,2)$. The variance is computed using Eq. (42). Note that the term proportional to $t^{2}$ in Eq. (63) will cancel out with the square of the first moment. Therefore, we get for the variance

$$
\kappa_{11}(t) \propto t, \quad \kappa_{22}(t) \propto t .
$$

The procedure here described will be adopted to calculate all the scalings of the first moment and the variance in the following.

At long times, i.e., in the asymptotic time regime, the PDF of transition times can be approximated with

$$
\hat{\psi}(\lambda) \approx 1-\tau_{0} \gamma\left(\tau_{g} \lambda\right)^{\delta}
$$

By substituting this expression into Eqs. (43) and (44), we get the scalings of the moments in the Laplace space. The mean value in the longitudinal direction behaves asymptotically as

$$
m_{1}^{*}(\lambda) \propto \lambda^{-1-\delta}
$$

By making use of the Tauberian theorems, we derive the asymptotic behavior of the variance in the temporal domain and we get

$$
m_{1}(t) \propto t^{\delta}
$$

This procedure is repeated to calculate all the scalings of the moments. In the transverse direction, as we have already pointed out before, the first moment in the transverse directions is always null $m_{2}=0$. For the spatial variances we obtain 


$$
\kappa_{11}(t) \propto t^{2 \delta}, \quad \kappa_{22}(t) \propto t^{\delta}
$$

\section{Strong Advective Heterogeneity}

We derive here the scalings of the moments for the case of strong advective heterogeneity. The latter is mapped onto heavy-tailed distributions of the mobile transition times. In particular, we will refer to inverse Gamma distributions for $\beta$ in $(1,2)$. Therefore, the Laplace transform of $\psi_{m}$ at long times can be approximated with

$$
\psi_{m}(\lambda) \approx 1-\alpha_{1} \lambda \tau_{v}+\alpha_{2}\left(\lambda \tau_{v}\right)^{\beta} .
$$

where $\alpha_{1}$ and $\alpha_{2}$ are constants. By inserting this expression into Eq. (18), we get the Laplace transform of the PDF of the compound process

$$
\psi^{*}(\lambda) \approx 1-\alpha_{1} \tau_{v}\left(\lambda+\gamma\left[1-p_{f}^{*}(\lambda)\right]\right)+\alpha_{2}\left(\tau_{v} \lambda+\tau_{v} \gamma\left[1-p_{f}^{*}(\lambda)\right]\right)^{\beta} .
$$

The Laplace transform of the distribution of trapping times at long times is approximated by Eq. (59). By substituting the latter into Eq. (70), we get the distribution of total transition times in the Laplace space

$$
\psi^{*}(\lambda) \approx 1-\alpha_{1}\left[\lambda \tau_{v}+\gamma \tau_{v}\left(\lambda \tau_{g}\right)^{\delta}\right]+\alpha_{2}\left[\lambda \tau_{v}+\gamma \tau_{v}\left(\lambda \tau_{g}\right)^{\delta}\right]^{\beta} .
$$

We now follow the same procedure described in "Weak Advective Heterogeneity" of Appendix to derive the scaling of the moments in the pre-asymptotic and asymptotic limit for different choices of the parameters $\beta$ and $\delta$. In the pre-asymptotic limit, the mean value of particle displacements scales linearly with time

$$
m_{1}(t) \propto t
$$

in the longitudinal direction, while in the transverse directions the mean is zero $m_{2}(t)=0$.

For the mean squared displacement, we find

$$
\kappa_{11}(t) \propto t^{3-\beta}
$$

in the direction of advection, while in the transverse direction the variance scales linearly

$$
\kappa_{22}(t) \propto t .
$$

Unlike the previous case, late behavior is strongly conditioned by the trapping properties of the medium and, as a consequence, the scalings of the moments will in general depend on the distribution of times that the particles spend in the immobile phase. In particular, the first moment in the longitudinal direction scales as

$$
m_{1}(t) \propto t^{\delta}
$$

while in the transverse directions the mean value is always null $m_{2}(t)=0$.

The scaling of the mean squared displacement along the direction in which advection occurs is given by

$$
\kappa_{11}(t) \propto t^{2 \delta},
$$

while in the transverse direction we get

$$
\kappa_{22}(t) \propto t^{\delta} .
$$




\section{Appendix 3: First Passage Time Distribution}

Here we will derive the scaling of the FPTD in the asymptotic and pre-asymptotic regimes for both weak and strong advective heterogeneity. The derivation will be performed for the same scenarios discussed in the previous section.

\section{Weak Advective Heterogeneity}

We will derive the expressions for the asymptotic regime. It has been previously shown that the distribution of transition times is given by Eq. (60). For $\lambda \tau_{e} \ll 1$, we obtain

$$
\psi^{*}(\lambda) \approx 1-\gamma \tau_{0}\left(\lambda \tau_{g}\right)^{\delta} .
$$

Inserting the latter into (50) and using (48) gives for the Laplace transform of the FPTD

$$
f^{*}\left(\lambda, x_{c}\right)=\exp \left[-\left\langle n_{c}\right\rangle \gamma \tau_{0}\left(\lambda \tau_{g}\right)^{\delta}\right],
$$

which is again a stable distribution characterized by the exponent $\delta$. This gives directly the scaling $f\left(t, x_{c}\right) \propto t^{-1-\delta}$ for $t \gg \tau_{e}$.

\section{Strong Advective Heterogeneity}

Inserting (71) in (50) gives for $f^{*}\left(\lambda, x_{c}\right)$

$$
f^{*}\left(\lambda, x_{c}\right) \approx \exp \left[-\left\langle n_{c}\right\rangle\left(\alpha_{1}\left[\lambda \tau_{v}+\gamma \tau_{v}\left(\lambda \tau_{g}\right)^{\delta}\right]+\alpha_{2}\left[\lambda \tau_{v}+\gamma \tau_{v}\left(\lambda \tau_{g}\right)^{\delta}\right]^{\beta}\right)\right] .
$$

For $\lambda \ll \gamma$, we approximate

$$
f^{*}\left(\lambda, x_{c}\right) \approx \exp \left[-\left\langle n_{c}\right\rangle \alpha_{1} \gamma \tau_{v}\left(\lambda \tau_{g}\right)^{\delta}\right]
$$

which gives $f\left(t, x_{c}\right) \propto t^{-1-\delta}$ for $t \gg \tau_{\gamma}$. For $\lambda \gg \gamma$, we have

$$
f^{*}\left(\lambda, x_{c}\right) \approx \exp \left(-\left\langle n_{c}\right\rangle\left[\alpha_{1} \lambda \tau_{v}+\alpha_{2}\left(\lambda \tau_{v}\right)^{\beta}\right]\right),
$$

which gives the pre-asymptotic scaling $f\left(t, x_{c}\right) \propto t^{-1-\beta}$ for $t \ll \tau_{\gamma}$.

\section{References}

Abramowitz, M., Stegun, I.A.: Handbook of Mathematical Functions. Dover, New York (1972)

Adams, E.E., Gelhar, L.W.: Field study of dispersion in a heterogeneous aquifer, 2. Spatial moment analysis. Water Resour. Res. 28(12), 3293-3308 (1992)

Barenblatt, G.I., Zheltov, I.P., Kochina, I.N.: Basic concepts in the theory of seepage of homogeneous liquids in fissured rocks [strata]. PMM 24(5), 825-864 (1960)

Barkai, E., Garini, Y., Metzler, R.: Strange kinetics of single molecules in living cells. Phys. Today 8(65), 29-35 (2012)

Barthelemy, P., Bertolotti, J., Wiersma, D.S.: A Lévy flight for light. Nature 453, 495-498 (2008)

Becker, M.W., Shapiro, A.M.: Interpreting tracer breakthrough tailing from different forced-gradient tracer experiment configurations in fractured bedrock. Water Resour. Res. 39, 1024 (2003)

Benke, R., Painter, S.: Modeling conservative tracer transport in fracture networks with a hybrid approach based on the Boltzmann transport equation. Water Resour. Res. 39, 1324 (2003)

Benson, D.A., Meerschaert, M.M.: A simple and efficient random walk solution of multi-rate mobile/immobile mass transport equations. Adv. Water Resour. 32(4), 532-539 (2009) 
Benson, D.A., Wheatcrat, S.W., Meerschaert, M.M.: Application of a fractional advection-dispersion equation. Water Resour. Res. 36, 1403-1421 (2000)

Berkowitz, B., Scher, H.: On characterization of anomalous dispersion in porous and fractured media. Water Resour. Res. 31(6), 1461-1466 (1995)

Berkowitz, B., Scher, H.: Anomalous transport in random fracture networks. Phys. Rev. Lett. 79(20), 40384041 (1997)

Berkowitz, B., Kosakowski, G., Margolin, G., Scher, H.: Application of continuous time random walk theory to tracer test measurements in fractured and heterogeneous porous media. Groundwater 39(4), 593-604 (2001)

Berkowitz, B., Cortis, A., Dentz, M., Scher, H.: Modeling non-fickian transport in geological formations as a continuous time random walk. Rev. Geophys. 44, RG2003 (2006)

Berkowitz, B., Emmanuel, S., Scher, H.: Non-fickian transport and multiple-rate mass transfer in porous media. Water Resour. Res. 44, W03402 (2008). doi:10.1029/2007WR005906

Bijeljic, B., Blunt, M.J.: Pore-scale modeling and continuous time random walk analysis of dispersion in porous media. Water Resour. Res. 42, W01202 (2006)

Bouchaud, J.P., Georges, A.: Anomalous diffusion in disordered media: statistical mechanisms, models and physical applications. Phys. Rep. 195(4,5), 127-293 (1990)

Carrera, J., Sánchez-Vila, X., Benet, I., Medina, A., Galarza, G., Guimerà, J.: On matrix diffusion: formulations, solution methods, and qualitative effects. Hydrol. J. 6, 178-190 (1998)

Cortis, A., Berkowitz, B.: anomalous transport in "classical" soil and sand columns. Soil Sci. Soc. Am. J. 68(1539-1548), 2004 (2004)

Cortis, A., Chen, Y., Scher, H., Berkowitz, B.: Quantitative characterization of pore-scale disorder effects on transport in "homogeneous" granular media. Phys. Rev. E 70, 041108 (2004)

Cushman, J.H., Ginn, T.R.: Fractional advection-dispersion equation: a classical mass balance with convolution-Fickian flux. Water Resour. Res. 36, 3763-3766 (2000)

Cushman, J.H., Hu, X., Ginn, T.R.: Nonequilibrium statistical mechanics of preasymptotic dispersion. J. Stat. Phys. 75(5/6), 859-878 (1994)

Cvetkovic, V., Fiori, A., Dagan, G.: Solute transport in aquifers of arbitrary variability: a time-domain random walk formulation. Water Resour Res. 50, WR015449 (2014)

de Anna, P., Le Borgne, T., Dentz, M., Tartakovsky, A.M., Bolster, D., Davy, P.: Flow intermittency, dispersion and correlated continuous time random walks in porous media. Phys. Rev. Lett. 110, 184502 (2013)

Dentz, M., Berkowitz, B.: Transport behavior of a passive solute in continuous time random walks and multirate mass transfer. Water Resour. Res. 39(5), 1111 (2003)

Dentz, M., Castro, A.: Effective transport dynamics in porous media with heterogeneous retardation properties. Geophys. Res. Lett. 36, L03403 (2009)

Dentz, M., Cortis, A., Scher, H., Berkowitz, B.: Time behavior of solute transport in heterogeneous media: transition from anomalous to normal transport. Adv. Water Resour. 27(2), 155-173 (2004)

Dentz, M., Gouze, P., Carrera, J.: Effective non-local reaction kinetics for transport in physically and chemically heterogeneous media. J. Contam. Hydrol. 120, 222-236 (2011)

Dentz, M., Gouze, P., Russian, A., Dweik, J., Delay, F.: Diffusion and trapping in heterogeneous media: an inhomogeneous continuous time random walk approach. Adv. Water Resour. 49, 13-22 (2012)

Dentz, M., Kang, P.K., Le Borgne, T.: Continuous time random walks for non-local radial solute transport. Adv. Water Resour. 82, 16-26 (2015)

Edery, Y., Guadagnini, A., Scher, H., Berkowitz, B.: Origins of anomalous transport in heterogeneous media: structural and dynamic control. Water Resour. Res. 50(2), 1490-1505 (2014)

Fiori, A., Jankovic, I., Dagan, G., Cvetkovic, V.: Ergodic transport trough aquifers of non-gaussian log conductivity distribution and occurence of anomalous behavior. Water Resour. Res. 43, W09407 (2007)

Gjetvaij, F., Russian, A., Gouze, P., Dentz, M.: Dual control of flow field heterogeneity and immobile porosity on non-fickian transport in berea sandstone. Water Resour. Res. (2015). doi:10.1002/2015WR017645

Gouze, P., Le Borgne, T., Leprovost, R., Lods, G., Poidras, T., Pezard, P.: Non-Fickian dispersion in porous media: 1. multi-scale measurements using single well injection withdrawal tracer tests at the ses sitjoles/aliance test site (Spain). Water Resour. Res. 44, W06426 (2008a)

Gouze, P., Melean, Z., Le Borgne, T., Dentz, M., Carrera, J.: Non-fickian dispersion in porous media explained by heterogeneous microscale matrix diffusion. Water Resour. Res. 44, W11416 (2008b)

Haggerty, R., Gorelick, S.M.: Multiple-rate mass transfer for modeling diffusion and surface reactions in media with pore-scale heterogeneity. Water Resour. Res. 31(10), 2383-2400 (1995)

Haggerty, R., McKenna, S.A., Meigs, L.C.: On the late time behavior of tracer test breakthrough curves. Water Resour. Res. 36(12), 3467-3479 (2000)

Haggerty, R.S., Fleming, S.W., Meigs, L.C., McKenna, S.A.: Tracer tests in a fractured dolomite: 2. Analysis of mass transfer in single-well injection-withdrawal tests. Water Resour. Res. 37, 1129-1142 (2001) 
Harvey, C.F., Gorelick, S.M.: Temporal moment-generating equations: modeling transport and mass transfer in heterogeneous aquifers. Water Resour. Res. 31(8), 1895-1911 (1995)

Hatano, Y., Hatano, N.: Dispersive transport of ions in column experiments: an explanation of long-tailed profiles. Water Resour. Res. 34(5), 1027-1033 (1998)

Holzner, M., Morales, V.L., Willmann, M., Dentz, M.: Intermittent lagrangian velocities and accelerations in three-dimensional porous medium flow. Phys. Rev. E 92, 013015 (2015)

Kang, P.K., Dentz, M., Le Borgne, T., Juanes, R.: Spatial markov model of anomalous transport through random lattice networks. Phys. Rev. Lett. 107, 180602 (2011)

Kang, P.K., de Anna, P., Nunes, J.P., Bijeljic, B., Blunt, M.J., Juanes, R.: Pore-scale intermittent velocity structure underpinning anomalous transport through 3-D porous media. Geophys. Res. Lett. 41(17), 6184-6190 (2014)

Kang, P.K., Le Borgne, T., Dentz, M., Bour, O., Juanes, R.: Impact of velocity correlation and distribution on transport in fractured media: field evidence and theoretical model. Water Resour. Res. 51, 940-959 (2015)

Kenkre, V.M., Montroll, E.W., Shlesinger, M.F.: Generalized master equations for continuous-time random walks. J. Stat. Phys. 9(1), 45-50 (1973)

Klafter, J., Sokolov, I.: Anomalous diffusion spreads its wings. Phys. World 18(8), 29-32 (2005)

Koch, D.L., Brady, J.F.: Anomalous diffusion in heterogeneous porous media. Phys. Fluids A 31, 965-1031 (1988)

Le Borgne, T., de Dreuzy, J.R., Davy, P., Bour, O.: Characterization of the velocity field organization in heterogeneous media by conditional correlation. Water Resour. Res. 43, 2006WR004875 (2007)

Le Borgne, T., Dentz, M., Carrera, J.: Spatial Markov processes for modeling Lagrangian particle dynamics in heterogeneous porous media. Phys. Rev. E 78, 041110 (2008a)

Le Borgne, T., Dentz, M., Carrera, J.: A Lagrangian statistical model for transport in highly heterogeneous velocity fields. Phys. Rev. Lett. 101, 090601 (2008b)

Levy, M., Berkowitz, B.: Measurement and analysis of non-Fickian dispersion in heterogeneous porous media. J. Contam. Hydrol. 64, 203-226 (2003)

Maloszewski, P., Zuber, A.: On the theory of tracer experiments in fissured rocks with a porous matrix. J. Hydrol. 79, 333 (1985)

Margolin, G., Dentz, M., Berkowitz, B.: Continuous time random walk and multirate mass transfer modeling of sorption. Chem. Phys. 295, 71-80 (2003)

Metzler, R., Jeon, J.H., Cherstvy, A.G., Barkai, E.: Anomalous diffusion models and their properties: nonstationary, non-ergodicity, and ageing at the centenary of single particle tracking. Phys. Chem. Chem. Phys. 16, 24128 (2014)

Michalak, A.M., Kitanidis, P.K.: Macroscopic behaviour and random-walk particle tracking of kinetically sorbing solutes. Water Resour. Res. 36, 2133-2146 (2000)

Montroll, E.W., Weiss, G.H.: Random walks on lattices, 2. J. Math. Phys. 6(2), 167 (1965)

Morales-Casique, E., Neuman, S.P., Guadagnini, A.: Nonlocal and localized analyses of nonreactive solute transport in bounded randomly heterogeneous porous media: theoretical framework. Adv. Water Resour. 29(8), 1238-1255 (2006)

Moroni, M., Cushman, J.H.: Statistical mechanics with three-dimensional particle tracking velocimetry experiments in the study of anomalous dispersion. II. Experiments. Phys. Fluids 13, 81-91 (2001)

Neuman, S., Zhang, Y.: A quasi-linear theory of non-fickian and fickian subsurface dispersion 1. Theoretical analysis with application to isotropic media. Water Resour. Res. 26(5), 887-902 (1990)

Roth, K., Jury, W.A.: Linear transport models for adsorbing solutes. Water Resour. Res. 29(4), 1195-1203 (1993)

Salamon, P., Fernàndez-Garcia, D., Gómez-Hernández, J.J.: Modeling mass transfer processes using random walk particle tracking. Water Resour. Res. 42, W11417 (2006)

Scher, H., Lax, M.: Stochastic transport in a disordered solid I. Theory. Phys. Rev. B 7(1), 4491-4502 (1973)

Scher, H., Margolin, G., Metzler, R., Klafter, J., Berkowitz, B.: The dynamical foundation of fractal stream chemistry: the origin of extremely long retention times. Geophys. Res. Lett. 29, 1061 (2002)

Schumer, R., Benson, D.A., Meerschaert, M.M., Bauemer, B.: Fractal mobile/immobile solute transport. Water Resour. Res. 39(10), 1296 (2003)

Villermaux, J.: Chemical engineering approach to dynamic modeling of linear chromatography, a simple method for representing complex phenomena from simple concepts. J. Chromatogr. 406, 11-26 (1987)

Weiss, G.H.: Aspects and Applications of the Random Walk. Elsevier, North-Holland (1994)

Willmann, M., Carrera, J., Sanchez-Vila, X.: Transport upscaling in heterogeneous aquifers: what physical parameters control memory functions? Water Resour. Res. 44, W12437 (2008)

Zhang, Y., Green, C.T., Baeumer, B.: Linking aquifer spatial properties and non-fickian transport in alluvial settings. J. Hydrol. 512, 315-331 (2014) 
Zinn, B., Meigs, L.C., Harvey, C.F., Haggerty, R., Peplinski, W.J., von Schwerin, C.F.: Experimental visualization of solute transport and mass transfer processes in two-dimensional conductivity fields with connected regions of high conductivity. Environ. Sci. Technol. 38, 3916-3926 (2004). doi:10.1021/es034958g 